\title{
SARS-CoV-2 antibody responses in patients with acute leukaemia
}

\author{
J. O'Nions $\mathbb{D}^{1} \cdot$ L. Muir $\mathbb{D}^{2} \cdot$ J. Zheng ${ }^{1} \cdot$ C. Rees-Spear $\mathbb{D}^{2} \cdot$ A. Rosa ${ }^{3} \cdot$ C. Roustan ${ }^{3} \cdot$ C. Earl $\mathbb{D}^{3} \cdot$ P. Cherepanov $^{3} \cdot$ \\ R. Gupta $\mathbb{1}^{1,4} \cdot$ A. Khwaja ${ }^{1,4} \cdot$ C. Jolly $\mathbb{1}^{2} \cdot$ L. E. McCoy $\mathbb{1}^{2}$
}

Received: 15 October 2020 / Revised: 4 November 2020 / Accepted: 17 November 2020 / Published online: 9 December 2020

(c) Springer Nature Limited 2020

\section{To the Editor:}

We read with interest the letter by Roeker et al., on SARSCoV-2 antibody responses in twenty-one patients with chronic lymphocytic leukemia (CLL), reporting that $67 \%$ generated antibodies to the SARS-CoV-2 Nucleoprotein antigen [1]. Their report highlighted the need to characterise the serological response to SAR-CoV-2 in immune compromised patients, particularly those with haematological malignancies, to help inform patient management and public health strategies. Patients with acute leukemia also represent a particularly high-risk group, who are reported to have some of the poorest outcomes of COVID-19 [2] and are hypothesised to have impaired immune SARS-CoV-2 responses due to either disease- or treatment-associated immune dysfunction. This has resulted in recommendations to reduce the risk of COVID-19 in these patients, including restructuring of clinical services, shielding and alterations in therapy to minimise their immune suppressive consequence [3]. Here we report SARS-CoV-2 antibody responses in a cohort of patients with acute leukemia and COVID-19 receiving systemic anti-cancer therapy (SACT) at

Supplementary information The online version of this article (https:// doi.org/10.1038/s41375-020-01103-2) contains supplementary material, which is available to authorized users.

J. O’Nions

jenny.onions@nhs.net

$\bowtie$ L. E. McCoy

1.mccoy@ucl.ac.uk

1 Department of Haematology, University College London NHS Foundation Trust, London, UK

2 Division of Infection and Immunity, University College London, London, UK

3 The Francis Crick Institute, London, UK

4 UCL Cancer Institute London, Paul O'Gorman Building, London, UK
University College London Hospital during the first wave of the pandemic.

Longitudinal serum samples were collected from nine patients with acute leukaemia, of whom eight had PCRconfirmed SARS-CoV-2 infection and one had a clinical diagnosis of COVD-19. Five patients had AML, three BALL and one T-ALL. Four patients commenced SACT prior to developing COVID-19 and five presented with leukemia and COVID-19. All patients received SACT within 28 days of developing COVID-19. Four patients received less myelosuppressive regimens (venetoclax azacitdine or gilteritinib) in accordance with NICE/NCRI COVID-19 guidance for acute leukaemia. COVID-19 symptoms were assigned from mild to severe [4], with two patients requiring ITU and mechanical ventilation. The median time between symptom onset and PCR diagnosis was 2.5 days (IQR 8.25), median duration of PCR positivity was 18.5 days (IQR 22) (Supplementary Fig. 1) and four patients received a potential COVID-19 modifying agent (tocilizumab, anakinra, remdesivir or dexamethasone). All patients survived and were discharged from hospital, with a median duration of illness of 30 days (IQR 30). Further patient demographics are described in Table 1.

Serum samples were taken a median of 9.5 days after positive PCR test for SARS-CoV-2 (range 1-25 days) and subsequent longitudinal serum samples taken between 2 and 103 days post onset of symptoms (POS). These were screened for anti-SARS-CoV-2 antibodies using ELISA to the external Spike glycoprotein (S1 subunit) and internal Nucleoprotein (N) [4-6]. Total serum IgG was within in the normal range in each case, excluding hypogammaglobinaemia. Seven of eight patients (88\%) with PCR-confirmed SARS-CoV-2 had IgG responses to $\mathrm{S} 1$ and $\mathrm{N}$ (Fig. 1a, b and appendix). Classifying patient samples into 7-day intervals POS (Supplementary Fig. 2) showed that seroconversion to $S$ appeared to precede $\mathrm{N}$, with only two patients seroconverting to both by day 30 (Supplementary Fig. 2 and Supplementary Tables 1-3). Overall seroconversion rates of $88 \%$ were similar to the general population $[4,6,7]$ and higher than that reported by 


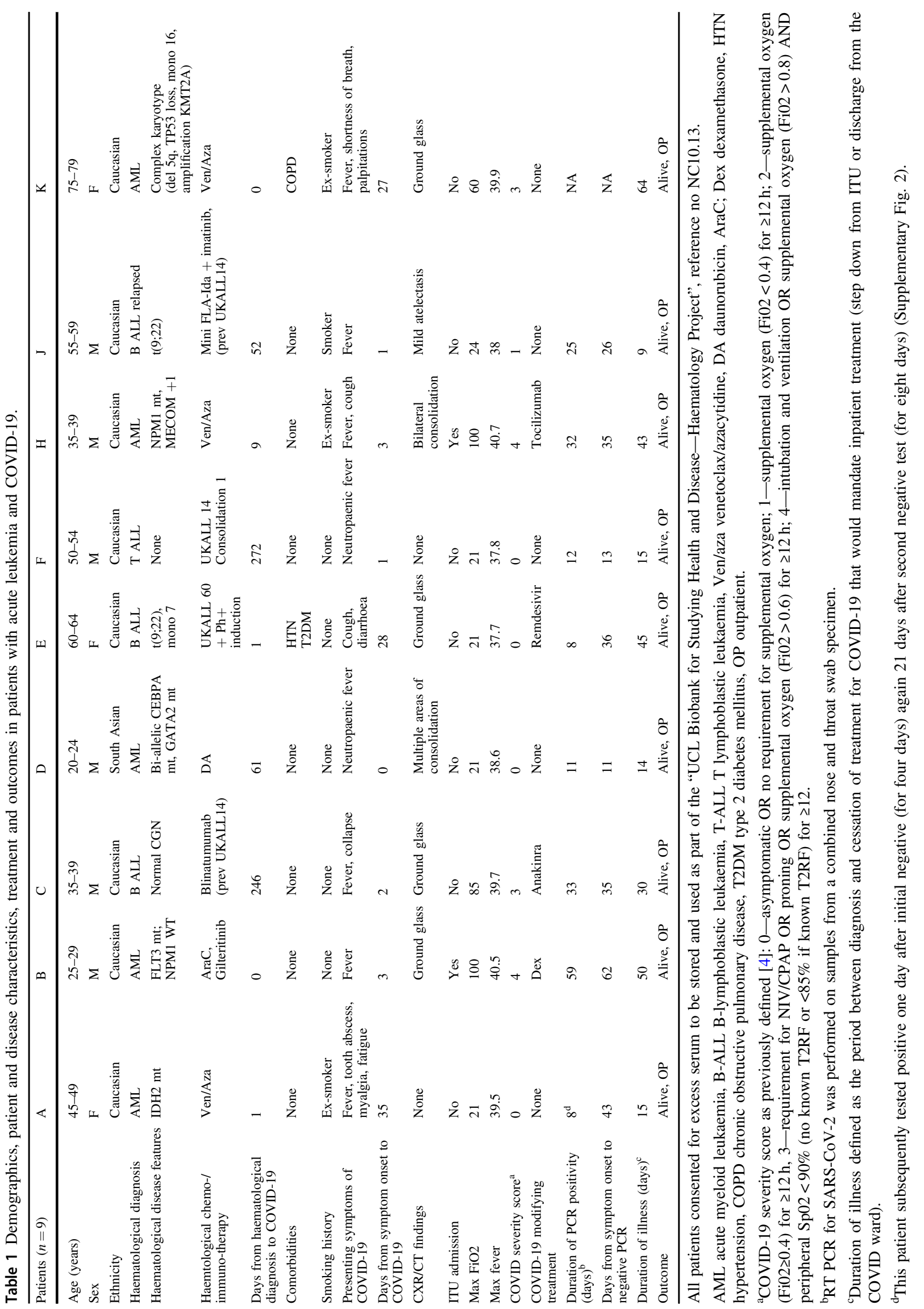


A
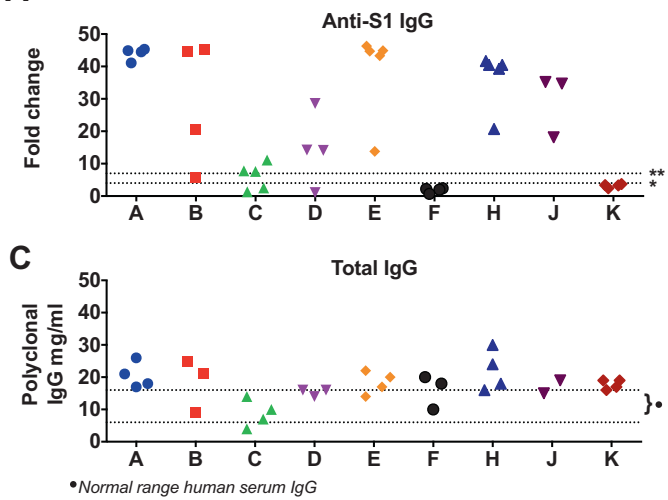

E
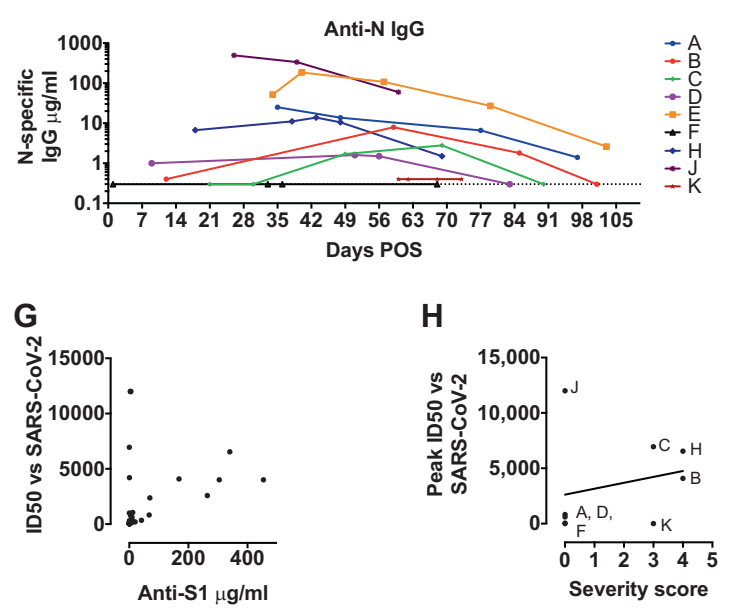

Fig. 1 Temporal dynamics and anti-viral function of SARS-CoV-2 antibody responses in acute leukaemia. Serum samples from patients A-K were assayed on ELISA plates pre-coated with S1 (a) or $\mathrm{N}$ (b). Antigen production and assay conditions were as previously described [4-6] except that all samples were treated with $0.5 \%$ NP40 before dilution in ELISA buffer. Absorbance was measured at $405 \mathrm{~nm}$ and data expressed as fold-change above blank background. The limit of detection (seropositivity) was determined as fold change $>4$ and is indicated by the dotted line marked $*$. The dotted line marked $* *$ indicates the limit of quantification in the assay depicted in $\mathbf{d}-\mathbf{e}$, determined by the linear range of the standard curve. c ELISA plates were coated overnight at $4{ }^{\circ} \mathrm{C}$ with goat anti-human $\mathrm{F}(\mathrm{ab})$ '2. Serum samples from patients $\mathrm{A}-\mathrm{K}$, and commercial polyclonal $\mathrm{IgG}$ standard, were titrated in ELISA buffer and added to the ELISA plate. Binding was detected with goat anti-human IgG conjugated to peroxidase and absorbance read at $450 \mathrm{~nm}$. IgG concentrations in serum were calculated based on interpolation from the IgG standard results using a fourparameter logistic (4PL) regression curve fitting model. Dotted lines marked with $\}^{*}$ indicate the normal average range for $\mathrm{IgG}$ in human serum. d, e Sera supplemented with $0.5 \%$ NP40 from patients A-K were diluted in ELISA buffer and then added to a blocked ELISA plates pre-coated with the indicated antigen and three lanes of goat

Roeker et al. for CLL [1]. Seroconversion appeared delayed in our cohort, with $50 \%$ seroconverting by day 28 , compared to $90 \%$ of healthy individuals [7], although this requries confirmation in a larger study. The temporal dynamics of SARS-CoV-2 responses in patients were measured using a
B

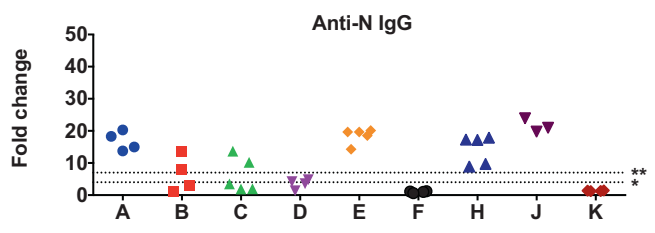

D

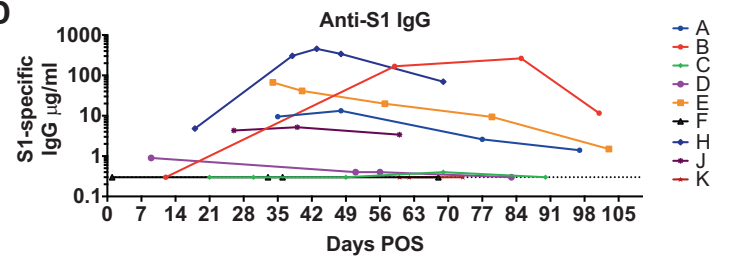

$\mathbf{F}$
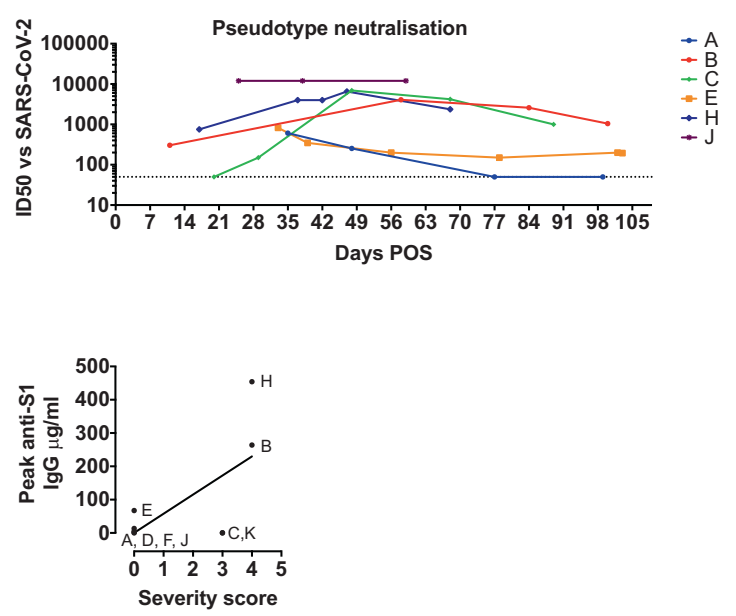

anti-human $\mathrm{F}(\mathrm{ab})$ '2 as per (c). Binding was detected with anti-IgG conjugated to alkaline phosphatase and absorbance measured at 405 $\mathrm{nm}$. Antigen-specific IgG concentrations in serum were calculated based on interpolation from the IgG standard results using a fourparameter logistic (4PL) regression curve fitting model. The dotted line indicates the limit of quantification, which is determined by the linear range of the standard curve and higher than the limit of detection in $\mathbf{a}$ and $\mathbf{b}$. f. Sequential serum samples from seropositive patients were titrated in duplicate and pre-incubated with luciferase-encoding HIV pseudotyped with the SARS-CoV-2 Spike for $1 \mathrm{~h}$ prior to the addition of HeLa cells expressing human ACE2 as previously described [7]. ID50 titres were only calculated in GraphPad Prism where at least two data points exhibited $>50 \%$ neutralisation. ID50 values for each patient are plotted on the $y$-axis against days POS on the $\mathrm{x}$-axis. g. ID50 values plotted against semi-quantitative $\mathrm{S} 1$ titres $(\mu \mathrm{g} / \mathrm{ml})$ for each sample. There is a trend to increasing ID50 with higher S1 titres but linear regression does not show a significant correlation $(r=0.06915$, $p=0.0925$ ). All data are from at least three independent experiments. h ID50 values and anti-S1 $(\mu \mathrm{g} / \mathrm{ml})$ values plotted against COVID-19 severity score for each patient as cited in Table 1. A trend line is shown but there were insufficient sample numbers to assess correlation and significance.

semi-quantitative assay of $\mathrm{S} 1$ and $\mathrm{N}$ antibodies. Again, the overall magnitude and persistence of SARS-CoV-2 IgG (up to $>100$ days POS) were similar to the general population. Furthermore, the patterns of antibody responses seen were as expected during an acute infection, namely; increasing titre 
reflective of seroconversion, declining titre suggestive of waning responses, and maintained titres (Fig. 1d, e). Similar kinetics of anti-N and anti-S1 antibody were seen, albeit with differences in overall antibody titre.

To address whether the antibodies generated by patients with acute leukemia were functional and able to inhibit SARS-CoV-2 infection, the capacity of serum to block viral infection in vitro was measured using an established pseudotyped SARS-CoV-2 neutralisation assay [7, 8] (Fig. 1f). Six of the seven $(86 \%)$ patients who seroconverted to SARS$\mathrm{CoV}-2$ generated antibodies able to inhibit virus infection, measured by determining the concentration of antibody capable of inhibiting SARS-CoV-2 infection by $50 \%$ (ID50\%). In general, neutralisation correlated with anti-S1 IgG levels (Fig. 1g); however, some patients showed strong neutralisation despite low anti-S1 titres, suggesting the presence of particularly potent antibodies similar to previous reports in individuals without cancer [7]. Importantly anti-S1 IgG titres and neutralising responses broadly correlated with clinical COVID-19 disease severity (Fig. 1h), although this correlation was not absolute with variation between individuals' responses observed. Again, this is in keeping with studies the general population and patients with haematological disease $[1,4,7]$.

Our finding that the majority of patients with acute leukemia on SACT are able to make antibody responses to SARS-CoV-2 has important implications for patient management, population-based serological monitoring and clinical decision making for this high-risk group. While our analysis of the kinetics, magnitude and function of SARS-CoV2 antibody responses in individuals with acute leukaemia align with similar SARS-CoV-2 serology studies on non-haematology patients [7], longitudinal sampling suggested a potential delay to SARS-CoV-2 seroconversion in patients with acute leukemia. This must be confirmed in a larger cohorts and in different subgroups of patients to determine if this is a feature of patients with haematological malignancy. Future studies should not only confirm but extend analysis of seroconversion rates, incorporating regular PCR testing to determine the precise onset and duration of SARS-CoV-2 infection, and frequent, longitudinal serum sampling to capture the emerging antibody response in greater depth. Notably, the ability to correlate antibody responses with function highlights the importance of measuring anti-S antibody responses alongside anti-N. Whether serological responses vary by leukaemia type or SACT regimen, and the influence this has on patient outcomes are essential next steps for investigation.

Acknowledgements We are extremely grateful to all the patients who participated in this study and the NHS staff that provided their clinical care. We would like to thank, Leo James and Jakub Luptak (LMB) for the provision of the plasmid encoding the $\mathrm{N}$ protein, and James E Voss
(TSRI) for providing the Hela-ACE2. LEM and LM are supported by an MRC Career Development Award (MR/R008698/1) to LEM. CJ is supported by a Wellcome Trust Investigator award (108079/Z/15/Z). RG acknowledges funding from Cure Cancer@ UCL.

Author contributions Designed the study: JO, LEM, CJ; Sample curation: JO, JZ, RG; Performed experiments: LM, LEM, CR-S; Generated reagents: AR, CR, CE, PC; Analysed data: JO, JZ, LM, LEM; Wrote/edited the manuscript: JO, LEM, CJ, LM, JZ, AK, RG.

\section{Compliance with ethical standards}

Conflict of interest The authors declare that they have no conflict of interest.

Ethical statement All clinical information was recorded and blood samples taken as routine standard of care. Patients were consented to allow any excess serum to be stored and used as part of the UCL Biobank for Studying Health and Disease, Haematology Project, reference no NC10.13, approved by the Leeds (East) Research Ethics Committee, UK.

Publisher's note Springer Nature remains neutral with regard to jurisdictional claims in published maps and institutional affiliations.

\section{References}

1. Roeker LE, Knorr DA, Pessin MS, Ramanathan LV, Thompson $\mathrm{MC}$, Leslie LA, et al. Anti-SARS-CoV-2 antibody response in patients with chronic lymphocytic leukemia. Leukemia. 2020;34: 3047-9.

2. Passamonti F, Cattaneo C, Arcaini L, Bruna R, Cavo M, Merli F, et al. Clinical characteristics and risk factors associated with COVID-19 severity in patients with haematological malignancies in Italy: a retrospective, multicentre, cohort study. Lancet Haematol. 2020;10:e737-45.

3. Zeidan AM, Boddu PC, Patnaik MM, Bewersdorf JP, Stahl M, Rampal RK, et al. Special considerations in the management of adult patients with acute leukaemias and myeloid neoplasms in the COVID-19 era: recommendations from a panel of international experts. Lancet Haematol. 2020;7:e601-12.

4. Pickering S, Betancor G, Galao RP, Merrick B, Signell AW, Wilson HD, et al. Comparative assessment of multiple COVID-19 serological technologies supports continued evaluation of point-ofcare lateral flow assays in hospital and community healthcare settings. PLoS Pathog. 2020;16:e1008817.

5. Houlihan CF, Vora N, Byrne T, Lewer D, Kelly G, Heaney J, et al. Pandemic peak SARS-CoV-2 infection and seroconversion rates in London frontline health-care workers. Lancet. 2020;396:e6-7.

6. Ng, KW, Faulkner N, Cornish G, Rosa A, Hussain S, Harvey R, et al. Pre-existing and de novo humoral immunity to SARS-CoV-2 in humans. Science. 2020. https://doi.org/10.1126/science.abe1107.

7. Seow J, Graham C, Merrick B, Acors S, Pickering S, Steel KJA, et al. Longitudinal observation and decline of neutralizing antibody responses in the three months following SARS-CoV-2 human infection. Nat Micro. 2020. https://doi.org/10.1038/s41564-02000813-8.

8. Brouwer PJM, Caniels TG, van der Straten K, Snitselaar JL, Aldon Y, Bangaru S, et al. Potent neutralizing antibodies from COVID-19 patients define multiple targets of vulnerability. Science. 2020;369: 643-50. 